\title{
Model Development for the Positioning Mechanisms in an Atomic Force Microscope
}

\author{
Ralph C. Smith \\ Center for Research in Scientific Computation \\ Department of Mathematics \\ North Carolina State University \\ Raleigh, NC 27695-8205 \\ rsmith@eos.ncsu.edu \\ Murti Salapaka \\ Electrical Engineering Department \\ Iowa State University \\ Ames, IA 50011 \\ murti@iastate.edu
}

\begin{abstract}
This paper addresses the development of distributed models for the piezoceramic positioning mechanisms employed in current atomic force microscope designs. Two common configurations for positioning mechanisms employ stacked actuators utilizing $d_{33}$ motion and piezoceramic shells utilizing a $d_{31}$ motion. In both cases, the relation between input voltages and generated displacements exhibits hysteresis and constitutive nonlinearities due to the ferroelectric nature of piezoceramic materials. Model development for these nanopositioners is considered in two steps. In the first, nonlinear constitutive relations that incorporate the hysteresis are developed through energy principles. In the second step, system models based on these constitutive equations are developed for the stacked actuator and piezoceramic shell. An abstract formulation encompassing both models is developed and used to establish well-posedness criteria. Numerical approximation techniques for the two models are summarized and the accuracy of the stacked actuator model is demonstrated through comparison with experimental data.
\end{abstract}




\section{Introduction}

Atomic force microscopes (AFM) provide the capability for obtaining angstrom-resolution measurements of both organic and inorganic samples by monitoring forces between the tip of a microcantilever employed in the microscope and atoms in the sample. To illustrate the principles and components of a prototypical AFM, consider the design depicted in Figure 1. The sample is moved both laterally and vertically beneath a highly flexible micro-cantilever by either a stage forced by a stacked piezoceramic (PZT) actuator or directly by a PZT shell. To ascertain the 3-D structure of the compound, the sample is moved along an $x-y$ grid using the lateral positioning mechanisms. As the sample moves, displacements in the cantilever tip are monitored using the photodiode and corresponding forces are determined via Hooke's law. The sample is then displaced in the $z$-direction to maintain constant forces with the displacement determined by a feedback law. A complete scan in this manner provides a surface image of the compound. Details regarding the construction and applications utilizing atomic force microscopes and scanning tunneling microscopes (STM) can be found in [10].

The degree of accuracy to which the PZT elements can laterally and vertically position the sample is crucial to the resolution of the final images. Two common positioning mechanisms employ stacked actuators utilizing $d_{33}$ motion and piezoceramic shells utilizing $d_{31}$ motion in response to input fields or voltages (see Figure 2). The stacked actuators offer the advantage of simple construction while generating the forces necessary for large scan positioning. The shell configurations are better suited for isolation from exogenous vibrations and small stage construction. While both configurations provide highly repeatable and accurate set point placement, the relations between input voltages and generated strains or displacements exhibit hysteresis and constitutive nonlinearities as illustrated in Figure 3 with data collected from an AFM employing a stacked actuator. At low frequencies, feedback mechanisms can adequately attenuate these effects thus leading to the success of the technologies. However, at the higher frequencies required for current and future applications, two phenomena degrade the accuracy achieved by present control techniques: (i) The piezoceramic materials exhibit increased hysteresis and (ii) The noise to data ratio increases to the point where feedback mechanisms are amplifying noise rather than attenuating unmodeled dynamics.

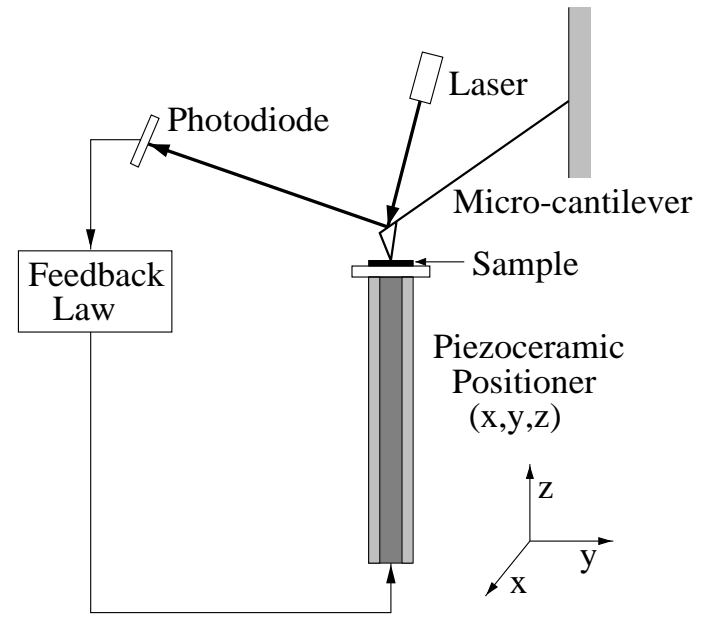

(a)

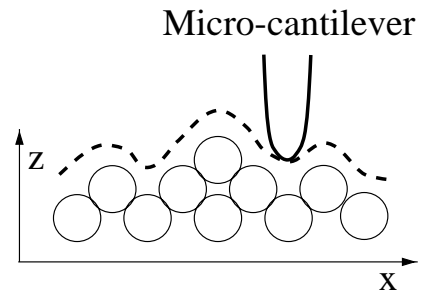

(b)

Figure 1: (a) Configuration of a prototypical AFM; (b) Surface image determined after one lateral sweep. 


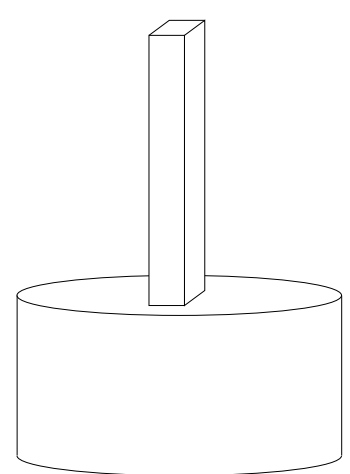

(a)

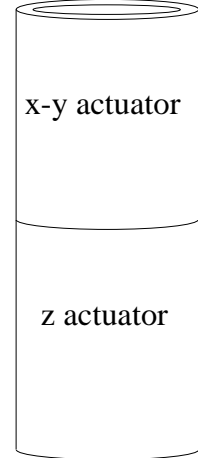

(b)

Figure 2: Actuator configurations employed for positioning in an atomic force microscope. (a) Rectangular stacked actuator; (b) Cylindrical transducer.

One approach being considered to address these problems and extend the technology to high frequency and broadband regimes is the incorporation of feedforward controllers utilizing model-based inverses to compensate for nonlinearities and hysteresis inherent to the positioning mechanisms. A crucial aspect of these control designs is the development of models for the positioning mechanisms which incorporate the nonlinear dynamics but are sufficiently low-order to permit real-time implementation. To achieve this efficiency, both low-order constitutive models and reduced-order system models are required.

In this paper, we develop system models for the stacked actuator and PZT shell depicted in Figure 2. Low-order constitutive relations are developed through the quantification of domain dynamics using energy principles. These nonlinear constitutive relations are then incorporated in classical rod and shell theory to obtain PDE models with nonlinear inputs. An abstract form which encompasses both models is developed and criteria required for model well-posedness are established. Numerical methods for discretizing the two models are summarized and the accuracy of the stacked actuator model is illustrated through a comparison with the experimental data plotted in Figure 3.

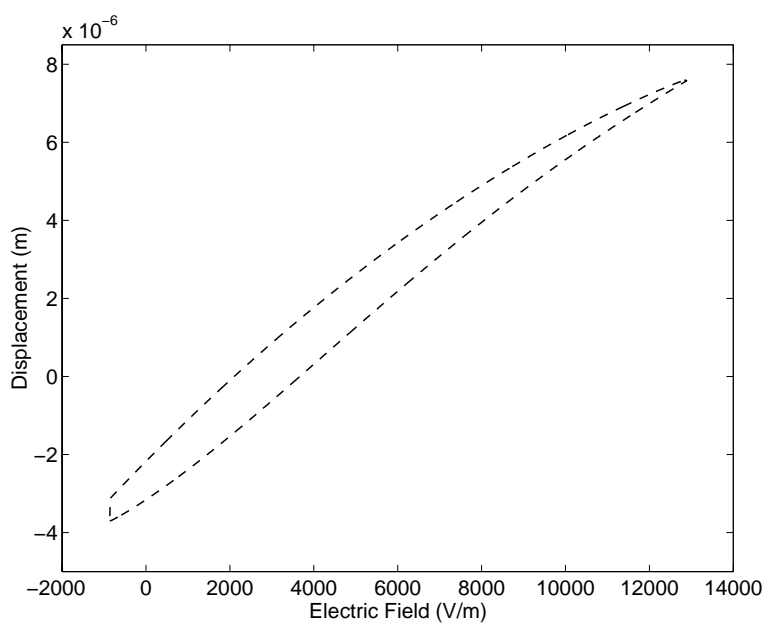

Figure 3: Relation between the input field $E$ and displacements generated by the PZT positioning mechanism in an AFM. 


\section{Constitutive Relations}

To establish constitutive relations for the PZT mechanisms, we make the assumption that the stressstrain behavior of the material is linear whereas the relation between the applied voltage $V$ or field $E$ and generated strains $e$ exhibits hysteresis and saturation nonlinearities as illustrated in Figure 3. Furthermore, we make the assumption that the relation between the polarization $P$ and strain is linear so that the hysteresis and nonlinear effects occur in the map $\mathcal{F}$ between the field and polarization. The assumption of linear relations between $P$ and $e$ is motivated by a number of classical references (e.g., $[5,11]$ ) and experiments are being designed to establish the validity of this assumption in the regimes under consideration. The nonlinearities and hysteresis in $\mathcal{F}$ have been experimentally validated for a number of PZT compounds [19] and these experiments have been used to evaluate the accuracy of various models for quantifying the nonlinear hysteresis map $\mathcal{F}$.

\section{$2.1 \quad 1-D$ Constitutive Relations}

In the absence of structural damping, constitutive nonlinearities, or hysteresis, it is illustrated in [11] that consideration of the elastic Gibbs free energy for piezoelectric materials yields the linear constitutive relations

$$
\begin{aligned}
& e=s^{P} \sigma+\beta P \\
& E=-b \sigma+\chi^{\sigma} P
\end{aligned}
$$

or equivalently

$$
\begin{aligned}
& \sigma=c^{P} e-c^{P} \beta P \\
& P=\chi \varepsilon_{0} E+\gamma \sigma .
\end{aligned}
$$

Here $e, \sigma, P$ and $E$ respectively denote the strain, stress, polarization and field while $s^{P}$ and $c^{P}=$ $1 / s^{P}$ are the compliance and Young's modulus at constant polarization values. Moreover, $b, \gamma$ and $\chi^{\sigma}=1 /\left(\chi \varepsilon_{0}\right)$ are piezoelectric and electric coupling coefficients, $\chi$ denotes the electric susceptibility, and $\varepsilon_{0}$ is the permittivity of free space. To express the actuator relation in (1) in terms of input voltages $V$, the linear polarizability relation $P=\chi \varepsilon_{0} E$ can be invoked along with the approximate formulation $V=E h$, where $h$ is the material thickness, to obtain

$$
\begin{aligned}
& \sigma=c^{P} e-c^{P} d V / h \\
& P=\chi \varepsilon_{0} E+\gamma \sigma .
\end{aligned}
$$

Two configurations for the piezoelectric coefficient $d=\beta \chi \varepsilon_{0}$ have been previously mentioned in the context of the stacked and cylindrical actuators. The stacked actuator utilizes a $d_{33}$ effect in which fields in the 3 direction generate displacements in the 3 direction. Alternatively, transverse displacements in the cylindrical transducer are generated by the $d_{31}$ effect in which fields through the shell thickness generate longitudinal (1-direction) displacements.

To incorporate internal damping, saturation nonlinearities, and hysteresis, we generalize (1) to obtain

$$
\begin{aligned}
\sigma & =c^{P} e+c_{D} \dot{e}-c^{P} \beta P(E, \sigma) \\
P & =\mathcal{F}(E, \sigma)
\end{aligned}
$$

where $c_{D}$ is the Kelvin-Voigt damping parameter and $\mathcal{F}$ quantifies the hysteresis and constitutive nonlinearities inherent to the materials. A number of techniques exist for determining appropriate maps $\mathcal{F}$ including Preisach characterizations [3, 9, 14, 22, 23] and domain models based on electrostatic energy principles $[18,19,20,21]$. The Preisach approach provides a rigorous mathematical 
characterization of hysteresis which is advantageous in regimes in which the underlying physics is poorly understood or difficult to quantify. However, the lack of an underlying energy principle leads to models requiring a large number of nonphysical parameters for the asymmetric responses arising in atomic force microscopy. It is also difficult in this approach to incorporate the frequency-dependence inherent to the materials. For these reasons, we employ domain wall models to specify the map $\mathcal{F}$.

As detailed in $[18,19]$, the relation between the input field and polarization $P$ is quantified in two steps. In the first, Boltzmann principles are employed to formulate the probability $\mu(\mathcal{E})=e^{-\mathcal{E} / k_{B} T}$ of attaining an electrostatic energy state $\mathcal{E}=-E \cdot p$ where $k_{B} T$ is the Boltzmann or kinetic energy of the dipoles $p$. Under the assumption that dipoles can align only in the direction of the effective field

$$
E_{e}(t)=E(t)+\alpha P(t)+E_{\sigma}(t),
$$

or opposite to it, summation of dipole contributions yields the Ising spin relation

$$
\widetilde{P}_{a n}(E(t), P(t), \sigma(t))=P_{s} \tanh \left(E_{e}(t) / a\right)
$$

for the anhysteretic polarization. Here $P_{s}$ denotes the saturation polarization, $a$ is a temperaturedependent normalization constant, $\alpha$ quantifies coupling effects due to neighboring dipoles, and $E_{\sigma}$ quantifies the field contributions due to stress-induced dipole rotation. The expression (4) is derived in the absence of bias fields and hence yields a symmetric characterization of certain domain switching mechanisms which produce certain saturation effects and hysteresis.

To quantify asymmetric minor loops due to biases in the field and polarization, it is necessary to modify the anhysteretic relation in the manner described in [17]. For a bias level $\left(E_{0}, P_{0}\right)$, due to field and polarization reversal at the point $\left(E_{1}, P_{1}\right)$, we employ the modified anhysteretic relation

$$
\begin{aligned}
& P(E, P, \sigma)=-\left[\Lambda \widetilde{P}_{a n}\left(2 E_{0}-E, 2 P_{0}-P, \sigma\right)-(1-\Lambda) \delta P_{s}\right]+2 P_{0} \\
& \Lambda=\frac{P_{1}+P_{s}}{\widetilde{P}_{a n}\left(E_{1}, P_{1}, \sigma\right)+P_{s}} .
\end{aligned}
$$

Here $\delta=\operatorname{sign}\left(\frac{d E}{d t}\right)= \pm 1$ and $\widetilde{P}_{a n}$ is given by (4).

Secondly, the reversible and irreversible energies associated with domain wall bending and translation are quantified by determining the energy required to reorient dipoles in the presence of the effective field $E_{e}$. As detailed in $[18,19]$, for low frequency drive regimes the resulting irreversible and reversible polarizations are respectively given by

$$
\frac{d P_{i r r}}{d t}=\frac{d E}{d t} \cdot \frac{\widetilde{\delta}\left(P_{a n}-P_{i r r}\right)}{k \delta-\alpha\left(P_{a n}-P_{i r r}\right)}
$$

and

$$
P_{\text {rev }}(t)=c\left(P_{a n}(t)-P_{\text {irr }}\right)(t)
$$

The total polarization is then quantified by the sum

$$
P(t)=P_{i r r}(t)+P_{r e v}(t) .
$$

The parameters $c, k, P_{s}, \alpha$ and $a$ are determined through either asymptotic relations or a least squares fit to data [19] while the parameter $\widetilde{\delta}=0$ or 1 is determined directly from field measurements. We note that for broadband or high frequency drive regimes, frequency-dependent models of the type discussed in [21] must be employed. 
For this model, the 1-D constitutive relations resulting from (2) are

$$
\begin{aligned}
& \sigma=c^{P} e+c_{D} \dot{e}-c^{P} \beta P(E, \sigma) \\
& P=c P_{a n}(E, P, \sigma)+(1-c) \int_{0}^{P_{i r r}} \frac{\widetilde{\delta}\left[P_{a n}(E, P, \sigma)-P_{i r r}(E, P, \sigma)\right]}{k \delta-\alpha\left[P_{a n}(E, P, \sigma)-P_{i r r}(E, P, \sigma)\right]} d P_{i r r}
\end{aligned}
$$

which, in the fully coupled form, are observed to be nonlinear due to the dependence of the polarization on stress as quantified by the term $E_{\sigma}$ in the effective field. For applications (e.g., self-sensing actuators) which utilize both the direct and converse piezoelectric effects, the coupling which produces the nonlinearity is important and must be retained. In purely actuator applications, such as for the AFM nanopositioner, one can obtain reasonable accuracy with $E_{\sigma}=0$. In this case, the system model resulting from (8) will be linear in the state with the hysteresis and other nonlinearities appearing only in the inputs. Finally, we note that while the integral formulation (8) is advantageous for certain theoretical issues, the formulation (7), with $P_{i r r}$ specified through numerical solution of the differential equation (6), is typically employed for material characterization and control design.

As a prelude to establishing model well-posedness for the atomic force microscope positioning mechanisms, we establish conditions which guarantee that the map between input fields and generated polarizations is well-defined. It is first necessary to restrict the admissible parameter set to guarantee that

$$
\left|k \delta-\alpha\left(P_{a n}-P_{i r r}\right)\right| \geq \varepsilon_{0}
$$

for $\delta= \pm 1$. This places a bound on the polarization curve which is reasonable from both mathematical and physical perspectives. Secondly, we define the set of turning points of $E$ in the manner specified in Definition 2.1. The existence of a unique solution to the constitutive model for $E_{\sigma} \equiv 0$ is established in Proposition 2.3. The more general case $E_{\sigma} \neq 0$ is less relevant for the initial analysis of this actuator application and is not addressed here.

Definition 2.1 Let $\mathcal{T}=\left\{t_{0}, t_{1}, \cdots, t_{n}\right\}$ denote the set of critical points of $E$ at which $\frac{d E}{d t}$ changes sign within the interval $[0, T]$.

Proposition 2.2 Assume that (9) is satisfied, $E \in C[0, T]$, and the partition $\mathcal{T}$ is specified. The differential equation (6) then has a unique solution on $[0, T]$.

Proof. Equation (6) has the form

$$
\frac{\partial P_{i r r}}{\partial t}=\mathcal{G}\left(P_{i r r}, t\right)
$$

where

$$
\mathcal{G}\left(P_{i r r}, t\right)=\frac{d E}{d t} \cdot \frac{\widetilde{\delta}\left(P_{a n}(t)-P_{i r r}\right)(t)}{k \delta-\alpha\left[P_{a n}(t)-P_{i r r}(t)\right]} .
$$

Since $\delta= \pm 1$ and $\widetilde{\delta}=0$ or 1 , it follows that on each subinterval $\left(t_{j}, t_{j+1}\right), \mathcal{G}$ and $\frac{\partial \mathcal{G}}{\partial P_{\text {irr }}}$ are continuous and bounded with

$$
\left|\frac{\partial \mathcal{G}}{\partial P_{\text {irr }}}\right| \leq \frac{k}{\varepsilon_{0}^{2}}
$$

From the theory of ordinary differential equations (e.g., see [12, Chapter 3]), it follows that (6) has a unique, continuously differentiable solution on each subinterval with well defined values at the endpoints $t_{j}$. Hence (6) has a unique solution $P_{i r r} \in C[0, T]$. 
Proposition 2.3 Consider the assumptions of Proposition 2.2. It then follows that

$$
P \in C[0, T]
$$

Proof. The result follows from the definitions of $P_{i r r}, P_{a n}$ and $P$.

\section{$2.2 \quad 2-D$ Constitutive Relations}

The 1-D constitutive relations (8) are appropriate for modeling the stacked actuator. However, they must be extended to 2-D to quantify the behavior of the cylindrical actuator.

For an applied field which produces $d_{31}$ motion, the extension of Hooke's law to a 2-D cylindrical domain yields the relations

$$
\begin{aligned}
& e_{x}=\frac{1}{c^{P}}\left(\sigma_{x}-\nu \sigma_{\theta}\right)+d_{31} E \\
& e_{\theta}=\frac{1}{c^{P}}\left(\sigma_{\theta}-\nu \sigma_{x}\right)+d_{31} E .
\end{aligned}
$$

Here $e_{x}, \sigma_{x}$ and $e_{\theta}, \sigma_{\theta}$ denote the strains and stresses in the longitudinal and circumferential directions and $\nu$ is the Poisson ratio. The inclusion of Kelvin-Voigt damping and formulation in terms of the voltage yields the linear constitutive relations

$$
\begin{aligned}
& \sigma_{x}=\frac{c^{P}}{1-\nu^{2}}\left(e_{x}+\nu e_{\theta}\right)+\frac{c_{D}}{1-\nu^{2}}\left(\dot{e}_{x}+\nu \dot{e}_{\theta}\right)-\frac{c^{P} d_{31}}{1-\nu} V \\
& \sigma_{\theta}=\frac{c^{P}}{1-\nu^{2}}\left(e_{\theta}+\nu e_{x}\right)+\frac{c_{D}}{1-\nu^{2}}\left(\dot{e}_{\theta}+\nu \dot{e}_{x}\right)-\frac{c^{P} d_{31}}{1-\nu} V \\
& P=d_{31} \sigma+\frac{\kappa^{\sigma} V}{h}
\end{aligned}
$$

The model (10) is appropriate if hysteresis and nonlinearities are negligible.

For regimes in which it is necessary to incorporate the inherent hysteresis and constitutive nonlinearities, the polarization-based relation (2) is generalized to obtain the nonlinear constitutive relations

$$
\begin{aligned}
& \sigma_{x}=\frac{c^{P}}{1-\nu^{2}}\left(e_{x}+\nu e_{\theta}\right)+\frac{c_{D}}{1-\nu^{2}}\left(\dot{e}_{x}+\nu \dot{e}_{\theta}\right)-\frac{c^{P} \beta}{1-\nu} P(E, \sigma) \\
& \sigma_{\theta}=\frac{c^{P}}{1-\nu^{2}}\left(e_{\theta}+\nu e_{x}\right)+\frac{c_{D}}{1-\nu^{2}}\left(\dot{e}_{\theta}+\nu \dot{e}_{x}\right)-\frac{c^{P} \beta}{1-\nu} P(E, \sigma) \\
& P(E, \sigma)=c P_{a n}(E, P, \sigma)+(1-c) \int_{0}^{P_{i r r}} \widetilde{\delta} \frac{P_{a n}-P_{i r r}}{k \delta-\alpha\left(P_{a n}-P_{i r r}\right)} d P_{i r r} .
\end{aligned}
$$

As in the 1-D case, state nonlinearities can be eliminated through the assumption that $E_{\sigma}=0$ in (3) which indicates that stresses have negligible effect on the polarization. For low drive regimes in which sensor (direct) piezoelectric effects are ignored, this assumption is reasonable and will be made in subsequent system models based on these constitutive relations. 


\section{System Models}

Two common actuator configurations for nanopositioning employ the stacked and cylindrical actuators depicted in Figure 2. We develop here system models for these configurations based on the nonlinear constitutive relations summarized in Section 2.

\subsection{Stacked Actuator}

When modeling the stacked actuator depicted in Figure 2a, it is assumed that the cross-sectional area is small as compared to the length so that only longitudinal motion is considered. This is consistent with the observation that the material is driven in a $d_{33}$ motion. For initial model development, we assume that the end of the actuator bonded to the AFM base is fixed and the opposite end is free. The model can be directly modified to accommodate mass or elastic loading at the free end.

The rod is assumed to have cross-sectional area $A$ and length $\ell$, and displacements in the longitudinal ( $x$-axis) are denoted by $u$. The density, Young's modulus and Kelvin-Voigt damping parameters are respectively denoted by $\rho, c^{P}$ and $c_{D}$.

Force balancing and the enforcement of boundary conditions then yields the strong form of the model

$$
\begin{aligned}
& \rho A \frac{\partial^{2} u}{\partial t^{2}}=\frac{\partial \mathcal{N}}{\partial x} \\
& u(t, 0)=\mathcal{N}(t, \ell)=0 .
\end{aligned}
$$

By employing the constitutive relation (8), along with the linear strain relation $e=\frac{\partial u}{\partial x}$, the force resultant $\mathcal{N}=\int_{A} \sigma d A$ for the rod is given by

$$
\mathcal{N}=c^{P} A \frac{\partial u}{\partial x}+c_{D} A \frac{\partial^{2} u}{\partial x \partial t}-c^{P} A \beta P(E)
$$

where the polarization is specified by (7) or (8). If the coupling term $E_{\sigma}$ is retained in the effective field relation (3), it is observed that the polarization is stress-dependent and hence (12) is fully nonlinear. However, under the assumption that $E_{\sigma}=0$, the nonlinearity occurs solely in the relation between the input field $E$ and the polarization $P$, and it is this regime that we consider.

To define a weak or variational form of the model which is appropriate for well-posedness analysis, approximation, or control design, the state $u$ is considered in the state space $X=L^{2}(0, \ell)$ with the inner product

$$
\langle\phi, \psi\rangle_{X}=\int_{0}^{\ell} \rho A \phi \psi d x
$$

The space of test functions is taken to be $V=H_{\ell}^{1}(0, \ell)=\left\{\psi \in H^{1}(0, \ell) \mid \psi(0)=0\right\}$ with the inner product

$$
\left\langle\left(c^{P}\right) \phi, \psi\right\rangle_{V}=\int_{0}^{\ell} c^{P} A \phi^{\prime} \psi^{\prime} d x .
$$

Multiplication by test functions followed by integration then yields the weak form

$$
\int_{0}^{\ell} \rho A \frac{\partial^{2} u}{\partial t^{2}} \psi d x+\int_{0}^{\ell}\left[c^{P} A \frac{\partial u}{\partial x}+c_{D} A \frac{\partial^{2} u}{\partial x \partial t}\right] \frac{\partial \psi}{\partial x}=-\int_{0}^{\ell} c^{P} A \beta P(E) \psi d x
$$

which must be satisfied for all $\psi \in V$. As detailed in Section 3.3, this provides a framework which facilitates the establishment of conditions which guarantee model well-posedness. 


\subsection{Cylindrical Actuators}

A second class of nanopositioning mechanisms are comprised of piezoceramic shells. We focus on the actuator employed for $z$-displacements since real-time control of this component is required to maintain constant forces between the sample and micro-cantilever. The mass of the shell employed for the $x-y$ translation is combined with the mass of the sample to provide an inertial force acting on the free end of the $z$-actuator.

For modeling purposes, we assume that the shell has length $\ell$, thickness $h$, and radius $R$. The axial direction is specified along the $z$-axis and the longitudinal, circumferential and transverse displacements are respectively denoted by $u, v$ and $w$. The density, Young's modulus and KelvinVoigt damping parameters are designated by $\rho, c^{P}, c_{D}$, and the region occupied by the middle surface of the shell is specified by $\Gamma_{0}=[0, \ell] \times[0,2 \pi]$. Finally, we consider the case in which the bottom edge of the shell $(x=0)$ is clamped and the opposite end $(x=\ell)$ is acted upon only by the inertial force associated with the combined mass of the $x-y$ actuator and the sample. For initial analysis involving longitudinal inputs and motion, the combined mass can be represented by a point mass $m$.

As detailed in $[4,13]$, force and moment balancing yield the Donnell-Mushtari shell equations

$$
\begin{aligned}
& R \rho h \frac{\partial^{2} u}{\partial t^{2}}-R \frac{\partial \mathcal{N}_{x}}{\partial x}-\frac{\partial \mathcal{N}_{x \theta}}{\partial \theta}=0 \\
& R \rho h \frac{\partial^{2} v}{\partial t^{2}}-\frac{\partial \mathcal{N}_{\theta}}{\partial \theta}-R \frac{\partial \mathcal{N}_{x \theta}}{\partial x}=0 \\
& R \rho h \frac{\partial^{2} w}{\partial t^{2}}-R \frac{\partial^{2} \mathcal{M}_{x}}{\partial x^{2}}-\frac{1}{R} \frac{\partial^{2} \mathcal{M}_{\theta}}{\partial \theta^{2}}-2 \frac{\mathcal{M}_{x \theta}}{\partial x \partial \theta}+\mathcal{N}_{\theta}=0
\end{aligned}
$$

where $\mathcal{N}_{x}, \mathcal{N}_{\theta}$ and $\mathcal{N}_{x \theta}$ are general force resultants and $\mathcal{M}_{x}, \mathcal{M}_{\theta}$ and $\mathcal{M}_{x \theta}$ are moment resultants. The boundary conditions at the fixed edge $x=0$ are taken to be

$$
u=v=w=\frac{\partial w}{\partial x}=0
$$

and the conditions

$$
\begin{array}{ll}
\mathcal{N}_{x}=-m \frac{\partial^{2} u}{\partial t^{2}} & , \mathcal{N}_{x \theta}+\frac{\mathcal{M}_{x \theta}}{R}=0 \\
\mathcal{Q}_{x}+\frac{1}{R} \frac{\partial \mathcal{M}_{x \theta}}{\partial \theta}=0 & , \quad \mathcal{M}_{x}=0
\end{array}
$$

are enforced at $x=\ell$. The first resultant condition incorporates the inertial force due to the mass of the piezoceramic cylinder employed for $y$ - $z$ translation along with the mass of the sample.

The force and moment resultants are specified by integrating the stress relations (11), or the product of the stress and moment arm, through the thickness of the shell. In the absence of shear stresses, this yields

$$
\begin{aligned}
& \mathcal{N}_{x}=\frac{c^{P} h}{1-\nu^{2}}\left(e_{x}+\nu e_{\theta}\right)+\frac{c_{D} h}{1-\nu^{2}}\left(\dot{e}_{x}+\nu \dot{e}_{\theta}\right)-\frac{c^{P} h \beta}{1-\nu} P(E) \\
& \mathcal{N}_{\theta}=\frac{c^{P} h}{1-\nu^{2}}\left(e_{\theta}+\nu e_{x}\right)+\frac{c_{D} h}{1-\nu^{2}}\left(\dot{e}_{\theta}+\nu \dot{e}_{x}\right)-\frac{c^{P} h \beta}{1-\nu} P(E) \\
& \mathcal{N}_{x \theta}=\frac{c^{P} h}{2(1+\nu)} e_{x \theta}+\frac{c_{D} h}{2(1+\nu)} \dot{e}_{x \theta}
\end{aligned}
$$


and

$$
\begin{aligned}
& \mathcal{M}_{x}=\frac{c^{P} h^{3}}{12\left(1-\nu^{2}\right)}\left(\kappa_{x}+\nu \kappa_{\theta}\right)+\frac{c_{D} h^{3}}{12\left(1-\nu^{2}\right)}\left(\dot{\kappa}_{x}+\nu \dot{\kappa}_{\theta}\right)-\frac{c^{P} h^{3} \beta}{12(1-\nu)} P(E) \\
& \mathcal{M}_{\theta}=\frac{c^{P} h^{3}}{12\left(1-\nu^{2}\right)}\left(\kappa_{\theta}+\nu \kappa_{x}\right)+\frac{c_{D} h^{3}}{12\left(1-\nu^{2}\right)}\left(\dot{\kappa}_{\theta}+\nu \dot{\kappa}_{x}\right)-\frac{c^{P} h^{3} \beta}{12(1-\nu)} P(E) \\
& \mathcal{M}_{x \theta}=\frac{c^{P} h^{3}}{24(1+\nu)} \tau+\frac{c_{D} h^{3}}{24(1+\nu)} \dot{\tau} .
\end{aligned}
$$

The midsurface strains and changes in curvature are given by

$$
\begin{array}{llll}
e_{x}=\frac{\partial u}{\partial x}, & e_{\theta}=\frac{1}{R} \frac{\partial v}{\partial \theta}+\frac{w}{R}, & e_{x \theta}=\frac{\partial v}{\partial x}+\frac{1}{R} \frac{\partial u}{\partial \theta} \\
\kappa_{x}=-\frac{\partial^{2} w}{\partial x^{2}} \quad, & \kappa_{\theta}=-\frac{1}{R^{2}} \frac{\partial^{2} w}{\partial \theta^{2}} \quad, & \tau=-\frac{2}{R} \frac{\partial^{2} w}{\partial x \partial \theta} .
\end{array}
$$

To reduce smoothness requirements on approximating solutions, it is again advantageous to consider a weak or variational form of the problem. To accomplish this, we consider the state space and space of test functions

$$
\begin{aligned}
& X=L^{2}\left(\Gamma_{0}\right) \times L^{2}\left(\Gamma_{0}\right) \times L^{2}\left(\Gamma_{0}\right) \\
& V=H_{\ell}^{1}\left(\Gamma_{0}\right) \times H_{\ell}^{1}\left(\Gamma_{0}\right) \times H_{\ell}^{2}\left(\Gamma_{0}\right)
\end{aligned}
$$

where $H_{\ell}^{1}\left(\Gamma_{0}\right)=\left\{\eta \in H^{1}\left(\Gamma_{0}\right) \mid \eta(0, \theta)=0\right\}$ and $H_{\ell}^{2}\left(\Gamma_{0}\right)=\left\{\eta \in H^{2}\left(\Gamma_{0}\right) \mid \eta(0, \theta)=\eta_{x}(0, \theta)=0\right\}$. For $\phi=(u, v, w)$ and $\psi=\left(\eta_{1}, \eta_{2}, \eta_{3}\right)$, the inner products are

$$
\langle\phi, \psi\rangle_{X}=\int_{\Gamma_{0}} \rho h u \bar{\eta}_{1} d \gamma+\int_{\Gamma_{0}} \rho h v \bar{\eta}_{2} d \gamma+\int_{\Gamma_{0}} \rho h w \bar{\eta}_{3} d \gamma+m u \bar{\eta}_{1}(\ell)
$$

and

$$
\begin{aligned}
\left\langle\left(c^{P}\right) \phi, \psi\right\rangle_{V} & =\int_{\Gamma_{0}} \frac{c^{P} h}{1-\nu^{2}}\left[\left(e_{x}+\nu e_{\theta}\right) \frac{\overline{\partial \eta_{1}}}{\partial x}+\frac{1}{2 R}(1-\nu) e_{x \theta} \frac{\overline{\partial \eta_{1}}}{\partial \theta}\right] d \gamma \\
& +\int_{\Gamma_{0}} \frac{c^{P} h}{1-\nu^{2}}\left[\left(e_{\theta}+\nu e_{x}\right) \frac{\overline{\partial \eta_{2}}}{\partial \theta}+\frac{1}{2 R}(1-\nu) e_{x \theta} \frac{\overline{\partial \eta_{2}}}{\partial x}\right] d \gamma \\
& +\int_{\Gamma_{0}} \frac{c^{P} h}{1-\nu^{2}}\left[\frac{1}{R}\left(e_{\theta}+\nu e_{x}\right) \bar{\eta}_{3}-\frac{h^{2}}{12}\left(\kappa_{x}+\nu \kappa_{\theta}\right) \frac{\overline{\partial^{2} \eta_{3}}}{\partial x^{2}}\right. \\
& \left.-\frac{h^{2}}{12 R^{2}}\left(\kappa_{\theta}+\nu \kappa_{x}\right) \frac{\overline{\partial^{2} \eta_{3}}}{\partial \theta^{2}}-\frac{h^{2}}{12 R}(1-\nu) \tau \frac{\partial^{2} \eta_{3}}{\partial x \partial \theta}\right] d \gamma
\end{aligned}
$$


A weak form of the model is then

$$
\begin{gathered}
\int_{\Gamma_{0}}\left\{R \rho h \frac{\partial^{2} u}{\partial t^{2}} \overline{\eta_{1}}+R N_{x} \frac{\overline{\partial \eta_{1}}}{\partial x}+N_{\theta x} \frac{\overline{\partial \eta_{1}}}{\partial \theta}-R \frac{c^{P} h \beta}{1-\nu} P(E) \frac{\partial \eta_{1}}{\partial x}\right\} d \gamma \\
-R m \frac{\partial^{2} u}{\partial t^{2}} \overline{\eta_{1}}(\ell)=0 \\
\int_{\Gamma_{0}}\left\{R \rho h \frac{\partial^{2} v}{\partial t^{2}} \overline{\eta_{2}}+N_{\theta} \frac{\overline{\partial \eta_{2}}}{\partial \theta}+R N_{x \theta} \frac{\overline{\partial \eta_{2}}}{\partial x}-\frac{c^{P} h \beta}{1-\nu} P(E) \frac{\overline{\partial \eta_{2}}}{\partial \theta}\right\} d \gamma=0 \\
\int_{\Gamma_{0}}\left\{R \rho h \frac{\partial^{2} w}{\partial t^{2}} \overline{\eta_{3}}+N_{\theta} \overline{\eta_{3}}-R M_{x} \frac{\overline{\partial^{2} \eta_{3}}}{\partial x^{2}}-\frac{1}{R} M_{\theta} \frac{\partial^{2} \eta_{3}}{\partial \theta^{2}}-2 M_{x \theta} \frac{\partial^{2} \eta_{3}}{\partial x \partial \theta}\right. \\
\left.+\frac{c^{P} h \beta}{1-\nu} P(E) \overline{\eta_{3}}+\frac{c^{P} h^{3} R \beta}{12(1-\nu)} P(E) \overline{\nabla^{2} \eta_{3}}\right\} d \gamma=0
\end{gathered}
$$

for all $\psi=\left(\eta_{1}, \eta_{2}, \eta_{3}\right) \in V$. The internal resultants $N_{x}=\mathcal{N}_{x}-\frac{c^{P} h \beta}{1-\nu} P(E), N_{\theta}=\mathcal{N}_{\theta}-\frac{c^{P} h \beta}{1-\nu} P(E)$, $N_{\theta x}=\mathcal{N}_{\theta x}, M_{x}=\mathcal{M}_{x}-\frac{c^{P} h^{3} \beta}{12(1-\nu)} P(E), M_{\theta}=\mathcal{M}_{\theta}-\frac{c^{P} h^{3} \beta}{12(1-\nu)} P(E)$ and $M_{\theta x}=\mathcal{M}_{\theta x}$ are comprised of the material contributions present in the absence of applied fields $E$. It is the model (19) that we consider when establishing criteria for model well-posedness and developing full and reduced-order approximation techniques.

\subsection{Abstract Model Formulation}

To provide a framework in which to establish model well-posedness, we consider an abstract formulation of the models based upon stiffness and damping sesquilinear forms. To this end, we define $\sigma_{i}: V \times V \rightarrow \mathbb{C}, i=1,2$, by

$$
\begin{aligned}
& \sigma_{1}(\phi, \psi)=\left\langle\left(c^{P}\right) \phi, \psi\right\rangle_{V} \\
& \sigma_{2}(\phi, \psi)=\left\langle\left(c_{D}\right) \phi, \psi\right\rangle_{V}
\end{aligned}
$$

where $V=H_{\ell}^{1}(0, \ell)$ with the inner product (14) for the rod model and $V=H_{\ell}^{1}\left(\Gamma_{0}\right) \times H_{\ell}^{1}\left(\Gamma_{0}\right) \times$ $H_{\ell}^{2}\left(\Gamma_{0}\right)$ with the inner product (18) for the cylindrical shell. In both cases, $\left\langle\left(c^{P}\right) \phi, \psi\right\rangle_{V}$ differs from $\left\langle\left(c_{D}\right) \phi, \psi\right\rangle_{V}$ only in that Young's moduli are replaced by Kelvin-Voigt damping parameters. It can be directly verified that the stiffness form $\sigma_{1}$ satisfies

$$
\begin{aligned}
& \left|\sigma_{1}(\phi, \psi)\right| \leq c_{1}|\phi|_{V}|\psi|_{V}, \text { for some } c_{1} \in \mathbb{R} \\
& \operatorname{Re} \sigma_{1}(\phi, \phi) \geq c_{2}|\phi|_{V}^{2}, \text { for some } c_{2}>0 \\
& \sigma_{1}(\phi, \psi)=\overline{\sigma_{1}(\psi, \phi)}
\end{aligned}
$$

for all $\phi, \psi \in V$. Moreover, the damping term $\sigma_{2}$ satisfies

$$
\begin{array}{ll}
\left|\sigma_{2}(\phi, \psi)\right| \leq c_{3}|\phi|_{V}|\psi|_{V}, \text { for some } c_{3} \in \mathbb{R} & \text { (Bounded) } \\
\operatorname{Re} \sigma_{2}(\phi, \phi) \geq c_{4}|\phi|_{V}^{2}, \text { for some } c_{4}>0 & (V \text {-Elliptic })
\end{array}
$$


For both the rod and shell models, the control input space is taken to be the Hilbert space $U=\mathbb{R}$. The input operators depend upon the specific model. For the rod model (15), $B: U \rightarrow V^{*}$ is defined by

$$
\langle[B(E)](t), \psi\rangle_{V^{*}, V}=[P(E)](t) \int_{0}^{\ell} c^{P} A \beta \frac{d \psi}{d x} d x
$$

whereas for the shell model (19), $B$ is defined by

$$
\begin{aligned}
\langle[B(E)](t), \psi\rangle_{V^{*}, V}=[P(E)](t) \int_{\Gamma_{0}} & \left\{R \frac{c^{P} h \beta}{1-\nu} \overline{\frac{\partial \eta_{1}}{\partial x}}+\frac{c^{P} h \beta}{1-\nu} \overline{\frac{\partial \eta_{2}}{\partial \theta}}\right. \\
+ & \left.\frac{c^{P} h \beta}{1-\nu} \overline{\eta_{3}}+\frac{c^{P} h^{3} \beta}{12(1-\nu) R} \overline{\nabla^{2} \eta_{3}}\right\} d \gamma
\end{aligned}
$$

In both cases, $\langle\cdot, \cdot\rangle_{V^{*}, V}$ denotes the usual duality product. It is observed that the operator $B$ can be expressed as

$$
[B(E)](t)=[b(E)](t) \cdot g \quad, \quad g \in V^{*}
$$

where

$$
\begin{aligned}
& {[b(E)](t)=c^{P} A \beta[P(E)](t)} \\
& g(\psi)=\int_{0}^{\ell} \psi^{\prime} d x
\end{aligned}
$$

for the rod and

$$
\begin{aligned}
& {[b(E)](t)=\frac{c^{P} \beta h}{1-\nu}[P(E)](t)} \\
& g(\psi)=\int_{\Gamma_{0}}\left\{R \frac{\overline{\partial \eta_{1}}}{\partial x}+\frac{\overline{\partial \eta_{1}}}{\partial \theta}+\overline{\eta_{3}}+\frac{h^{2}}{(1+\nu) 12 R} \overline{\nabla^{2} \eta_{3}}\right\} d \gamma
\end{aligned}
$$

for the shell.

The weak form of the rod model (15) or shell model (19) can then be written in the abstract variational form

$$
\langle\ddot{y}(t), \psi\rangle_{V^{*}, V}+\sigma_{2}(\dot{y}(t), \psi)+\sigma_{1}(y(t), \psi)=\langle[B(E)](t), \psi\rangle_{V^{*}, V}
$$

for all $\psi \in V$. For the rod model, $y=u, V=H_{\ell}^{1}(0, \ell)$, the duality product is the extension of the $X$-inner product defined by (13) and $B$ is defined by (21). For the shell model, $y=(u, v, w), B$ is defined in (22), $V=H_{\ell}^{1}\left(\Gamma_{0}\right) \times H_{\ell}^{1}\left(\Gamma_{0}\right) \times H_{\ell}^{2}\left(\Gamma_{0}\right)$, and the duality product is the extension of (17).

Alternatively, one can define the operators $A_{i} \in \mathcal{L}\left(V, V^{*}\right), i=1,2$, by

$$
\left\langle A_{i} \phi_{1}, \phi_{2}\right\rangle_{V^{*}, V}=\sigma_{i}\left(\phi_{1}, \phi_{2}\right)
$$

and formulate the model in operator form as

$$
\ddot{y}(t)+A_{2} \dot{y}(t)+A_{1} y(t)=[B(E)](t)
$$

in the dual space $V^{*}$. Well-posedness criteria are established in the following lemma and theorem.

Lemma 3.1 Define the operator B by (21) or (22) and let $\mathcal{T}$ denote the partition specified in Definition 2.1. Under the assumption that $E \in C[0, T]$ and that the parameters satisfy (9), it follows that

$$
B(E) \in L^{2}\left((0, T) ; V^{*}\right)
$$


Proof. From (23), the operator $B$ can be expressed as $[B(E)](t)=[b(E)](t) \cdot g$ where $g \in V^{*}$ and $b$ are defined in (24) or (25). It follows from Proposition 2.3 that

$$
b(\cdot): C[0, T] \rightarrow C[0, T]
$$

so that the norm

$$
\|[B(E)](t)\|_{V^{*}}=\sup _{v \in V} \frac{|[b(E)](t) \cdot g(v)|}{\|v\|_{V}}
$$

exists for each $t \in[0, T]$. Since $\|[B(E)](t)\|_{V^{*}}=|[b(E)](t)| \cdot\|g\|_{V^{*}}$, it follows that

$$
\|B(E)\|_{L^{2}\left((0, T) ; V^{*}\right)}^{2} \leq \max _{t \in[0, T]}\left\{|[b(E)](t)|^{2}\right\} \cdot T \cdot\|g\|_{V^{*}}^{2}
$$

which implies that

$$
B(E) \in L^{2}\left((0, T) ; V^{*}\right)
$$

Theorem 3.2 Let $\sigma_{1}$ and $\sigma_{2}$ be given by (20) and consider the assumptions of Lemma 3.1. There then exists a unique solution y to (26) which satisfies

$$
\begin{aligned}
& y \in C((0, T) ; V) \\
& \dot{y} \in C((0, T) ; X) .
\end{aligned}
$$

The result follows directly from Theorem 4.1 of [4] or Theorem 2.1 and Remark 2.1 of [2].

\section{Numerical Approximation Techniques}

To implement the models for either the rectangular stacked actuator or the cylindrical actuator, it is necessary to develop appropriate approximation techniques to discretize the modeling PDE. To accomplish this, we consider general Galerkin methods in which basis functions are comprised of spline or spline-Fourier tensor products. The resulting methods can accommodate a variety of boundary conditions, are sufficiently accurate to resolve fine-scale dynamics, and can be employed for constructing reduced-order POD approximates for real-time implementation.

\subsection{Stacked Actuator Model}

To approximate the weak form of the stacked actuator model (15), we employ a finite element discretization in space followed by a finite difference discretization in time. The semidiscrete system resulting from the finite element approximation is appropriate for finite dimensional, continuous time control design whereas the fully discrete system is amenable to simulations and control implementation.

To obtain a semidiscrete system, we consider a uniform partition of $[0, \ell]$ with points $x_{i}=i h$, $i=0,1, \cdots, N$ with step size $h=\ell / N$ where $N$ denotes the number of subintervals. The spatial 
basis $\left\{\psi_{i}\right\}_{i=1}^{N}$ is then comprised of of linear splines

$$
\begin{aligned}
& \psi_{i}(x)=\frac{1}{h}\left\{\begin{array}{l}
\left(x-x_{i-1}\right), x_{i-1} \leq x<x_{i} \\
\left(x_{i+1}-x\right), x_{i} \leq x \leq x_{i+1} \\
0, \text { otherwise }
\end{array} \quad, \quad i=1, \cdots, N-1\right. \\
& \psi_{N}(x)=\frac{1}{h}\left\{\begin{array}{l}
\left(x-x_{N-1}\right), x_{N-1} \leq x \leq x_{N} \\
0, \text { otherwise }
\end{array}\right.
\end{aligned}
$$

(see [16] for details regarding the convergence analysis for the method). The solution $u(t, x)$ to $(15)$ is then approximated by the expansion

$$
u^{N}(t, x)=\sum_{j=1}^{N} u_{j}(t) \psi_{j}(x)
$$

Because $V^{N}=\operatorname{span}\left\{\psi_{i}\right\}_{i=1}^{N} \subset V=H_{\ell}^{1}(0, \ell)$, the approximate solution satisfies the essential boundary condition $u^{N}(t, 0)=0$ and can attain arbitrary displacements at $x=\ell$.

The projection of the problem (15) onto the finite dimensional subspace $V^{N}$ yields the semidiscrete system

$$
\begin{aligned}
& \dot{y}(t)=A y(t)+[F(E)](t) \\
& y(0)=y_{0}
\end{aligned}
$$

where $y(t)=\left[u_{1}(t), \cdots, u_{N}(t), \dot{u}_{1}(t), \cdots, \dot{u}_{N}(t)\right]^{T}$ and

$$
A=\left[\begin{array}{cc}
0 & I \\
-Q^{-1} K & -Q^{-1} C
\end{array}\right] \quad, \quad[F(E)](t)=\left[\begin{array}{c}
0 \\
Q^{-1}[f(E)](t)
\end{array}\right] .
$$

The mass, stiffness, damping and forcing matrices have the components

$$
\begin{aligned}
& {[Q]_{i j}=\int_{0}^{\ell} \rho A \psi_{i} \psi_{j} d x \quad,[f(E)]_{i}(t)=[P(e)](t) \int_{0}^{\ell} c^{P} A \beta \psi_{i}^{\prime} d x} \\
& {[K]_{i j}=\int_{0}^{\ell} c^{P} A \psi_{i}^{\prime} \psi_{j}^{\prime} d x \quad, \quad[C]_{i j}=\int_{0}^{\ell} c_{D} A \psi_{i}^{\prime} \psi_{j}^{\prime} d x .}
\end{aligned}
$$

The system (27) can be employed for finite-dimensional control design. For subsequent implementation, we consider a temporal discretization of (27) using a modified trapezoid rule. For temporal stepsizes $\Delta t$, this yields the difference equations

$$
\begin{aligned}
& y_{j+1}=\mathcal{A} y_{j}+[\mathcal{B}(E)]\left(t_{j}\right) \\
& y_{0}=y(0)
\end{aligned}
$$

where $t_{j}=j \Delta t, y_{j}$ approximates $y\left(t_{j}\right)$, and

$$
\mathcal{A}=\left[I-\frac{\Delta t}{2} A\right]^{-1}\left[I+\frac{\Delta t}{2} A\right] \quad, \quad[\mathcal{B}(E)]\left(t_{j}\right)=\Delta t\left[I-\frac{\Delta t}{2} A\right]^{-1}[F(E)]\left(t_{j}\right) .
$$

This yields an A-stable, single step method requiring moderate storage and providing moderate accuracy. 


\subsection{Cylindrical Actuator Model}

Due to the inherent coupling between longitudinal, circumferential, and transverse displacements in combination with the 2-D support of the middle surface, the numerical approximation of the model for the cylindrical actuator is significantly more complicated than the approximation of the stacked actuator model. Among the issues which must be addressed when constructing finite element or general Galerkin methods for the shell is the choice of elements which avoid shear and membrane locking and the maintenance of boundary conditions. We summarize here a spline-based Galerkin method developed in [6] for thin shells and direct the reader to that source for details regarding the construction of constituent matrices and convergence properties of the method. Details regarding the use of this approximation method for LQR control of shells utilizing piezoceramic actuators can be found in $[7,8]$.

The bases for the $u, v$ and $w$ displacements are respectively taken to be $\mathcal{B}_{u_{k}}(\theta, x)=e^{i m \theta} B_{u_{j}}(x)$, $\mathcal{B}_{v_{k}}(\theta, x)=e^{i m \theta} B_{v_{j}}(x)$, and $\mathcal{B}_{w_{k}}(\theta, x)=e^{i m \theta} B_{w_{j}}(x)$ where $B_{u_{j}}, B_{v_{j}}$ and $B_{w_{j}}$ are cubic $B$ splines modified to satisfy the boundary conditions (e.g., see page 79 of [16]). The approximating subspace is $V^{\mathcal{N}}=\operatorname{span}\left\{\mathcal{B}_{u_{k}}\right\} \times \operatorname{span}\left\{\mathcal{B}_{v_{k}}\right\} \times \operatorname{span}\left\{\mathcal{B}_{w_{k}}\right\}$ and displacements are approximated through the expansions

$$
\begin{aligned}
u^{\mathcal{N}}(t, \theta, x) & =\sum_{k=1}^{\mathcal{N}_{u}} u_{k}(t) \mathcal{B}_{u_{k}}(\theta, x) \\
v^{\mathcal{N}}(t, \theta, x) & =\sum_{k=1}^{\mathcal{N}_{v}} v_{k}(t) \mathcal{B}_{v_{k}}(\theta, x) \\
w^{\mathcal{N}}(t, \theta, x) & =\sum_{k=1}^{\mathcal{N}_{w}} w_{k}(t) \mathcal{B}_{w_{k}}(\theta, x) .
\end{aligned}
$$

The restriction of the problem (19) to $V^{\mathcal{N}}$ and construction of the forcing vectors then yields the matrix system

$$
\begin{aligned}
& \dot{y}(t)=A y(t)+[F(E)](t)+G(t) \\
& y(0)=y_{0}
\end{aligned}
$$

where $y(t)=[\vartheta(t), \dot{\vartheta}(t)]^{T} \in \mathbb{R}^{2 \mathcal{N}}, \vartheta(t)=\left[u_{1}(t), \cdots, u_{\mathcal{N}_{u}}(t), v_{1}(t), \cdots, v_{\mathcal{N}_{v}}(t), w_{1}(t), \cdots, w_{\mathcal{N}_{w}}(t)\right]^{T}$, $\mathcal{N}=\mathcal{N}_{u}+\mathcal{N}_{v}+\mathcal{N}_{w}$, denotes the coefficients and their derivatives. The matrix $A$ and vector $F$ have the general form $(28)$ and the vector $G(t)=\left[0, Q^{-1} g(t)\right]^{T}$ incorporates the boundary contributions due to the inertial mass and applied load at the end of the cylinder. The reader is referred to [6] for details concerning the construction of the mass, stiffness and damping matrices $Q, K$ and $C$.

\section{Model Validation}

To illustrate the attributes and capabilities of the models, we consider the characterization of the stacked actuator depicted in Figure 2a. To accommodate the dimensions and longitudinal dynamics exhibited by the actuator, the rod model (15) resulting from the nonlinear constitutive relation (8) is employed. For numerical implementation, we employ the resulting discrete system (29).

The stacked actuator has a width and thickness of $5 \mathrm{~mm}$ and length of $20 \mathrm{~mm}$. The Young's modulus and density specified by the manufacturer are $c^{P}=6.5 \times 10^{10} \mathrm{~N} / \mathrm{m}^{2}$ and $\rho=7730 \mathrm{~kg} / \mathrm{m}^{3}$. The values $c_{D}=6.5 \times 10^{7} \mathrm{Ns} / \mathrm{m}^{2}$ and $\beta=3.52 \times 10^{-2}$ for the Kelvin-Voigt damping parameter and piezoelectric coupling coefficient were estimated through a least squares fit to the data. Finally, the asymptotic relations summarized in [19] were employed in combination with a least squares algorithm 


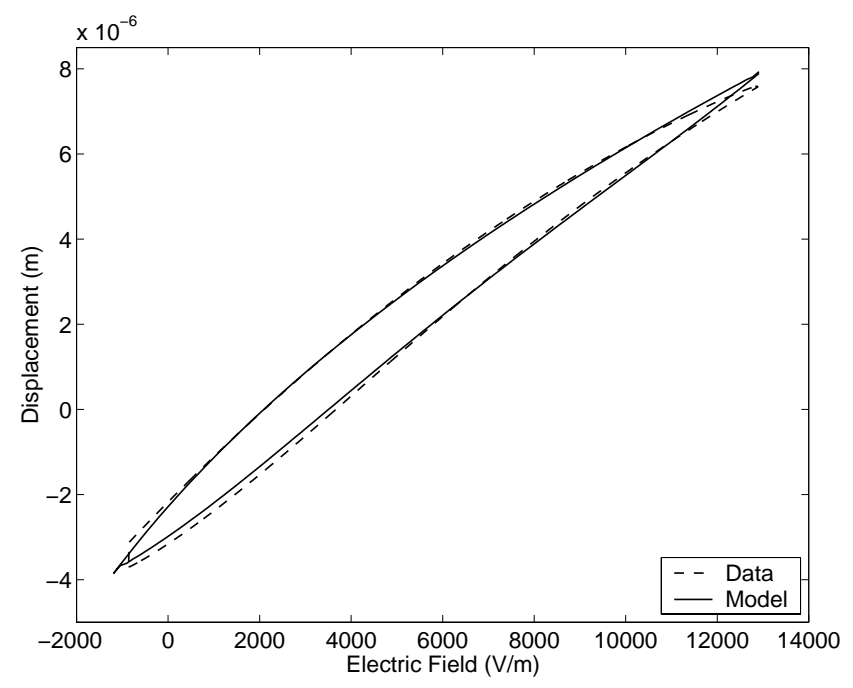

Figure 4: Experimental stacked actuator data and model prediction.

to obtain the parameters $\alpha=4.5 \times 10^{6} \mathrm{Vm} / \mathrm{C}, a=4.6 \times 10^{5} \mathrm{C} / \mathrm{m}^{2}, k=1.4 \times 10^{5} \mathrm{C} / \mathrm{m}^{2}, c=.81$ and $P_{s}=.49 \mathrm{C} / \mathrm{m}^{2}$ for the hysteresis component of the model.

The model prediction obtained with these parameters is compared with the actuator data in Figure 4. It is observed that the model accurately quantifies both the constitutive nonlinearities and hysteresis inherent to this nanopositioning mechanism to a degree which is sufficiently accurate for both device characterization and the construction of inverse compensators for subsequent control design.

Furthermore, it was observed that because longitudinal piezoelectric inputs are manifested as stress resultants at the end of the rod, there is little spatial variation for the considered configuration. Hence convergence of the finite element method was attained by $N=8$ with only moderate discrepancies produced with fewer basis functions. The ramifications of this are twofold: (i) the method is very fast which facilitates real-time implementation, and (ii) sufficient accuracy for certain drive regimes may be attained with lumped ODE models for the rod. The quantification of conditions under which ODE rod models may be applicable is under investigation. It is noted, however, that such simplifications will not be feasible for the shell model used to characterize the cylindrical actuator due to the inherent coupling between component displacements. Hence the full PDE model or appropriate reduced-order models will be required for all configurations utilizing cylindrical actuators.

\section{Concluding Remarks}

The PDE models developed here provide a means of characterizing two of the actuator configurations employed in current atomic force microscopes and projected nanopositioning designs. As illustrated through data from the stacked actuator, hysteresis and constitutive nonlinearities inherent to the constituent piezoelectric materials are manifested even at the low drive levels required for nanopositioning. It is crucial that these nonlinear mechanisms be accommodated in device characterization and subsequent control design to achieve the angstrom-level resolution required from the actuators.

At low drive frequencies, the deleterious effects of the hysteresis and nonlinearities can be mitigated through feedback mechanisms thus leading to the phenomenal success of the instruments. To 
achieve the high frequency scan rates required for future applications, however, inverse compensators based on low-order models in conjunction with feedforward control designs will be required to achieve the necessary accuracy.

Two crucial attributes of models considered for use in inverse compensator design are the properties that they can be inverted and are sufficiently low-order to permit real-time implementation. As illustrated [15], models of the type presented here are amenable to either full inversion, through the solution of complementary ODE, or partial inversion through consideration of the anhysteretic component of the model. The design and implementation of feedforward control designs which utilize the resulting inverse compensators is under investigation. As discussed in Section 5, the rod model exhibits minimal spatial variation in certain drive regimes which indicates that lumped parameter ODE models may be adequate for certain nanopositioner designs. For regimes in which significant spatial variation necessitates the retention of the full PDE, as well as the characterization of nanopositioners employing cylindrical actuators, the development of reduced-order models will be necessary to achieve real-time, broadband implementation. We are currently pursuing the development of actuator models based on proper orthogonal decomposition (POD) expansions due to recent success utilizing this reduced-order approach in control design for cylindrical shells with surface mounted piezoceramic actuators [1]. The resulting reduced models employing inverse algorithms will then be considered for broadband control design in nanopositioning applications.

\section{Acknowledgements}

This research was supported in part by the Air Force Office of Scientific Research under the grant AFOSR-F49620-01-1-0107 and the National Science Foundation under the grant CMS-0099764.

\section{References}

[1] H.T. Banks, R.C.H. del Rosario and R.C. Smith, Reduced Order Model Feedback Control Design: Numerical Implementation in a Thin Shell Model, IEEE Transactions on Automatic Control, 45(7) (2000), pp. 1312-1324.

[2] H.T. Banks, K. Ito and Y. Wang, Well-Posedness for Damped Second Order Systems with Unbounded Input Operators, Differential and Integral Equations, 8 (1995), pp. 587-606.

[3] H.T. Banks, A.J. Kurdila and G. Webb, Identification of Hysteretic Control Influence Operators Representing Smart Actuators Part I: Formulation, Mathematical Problems in Engineering, 3 (1997), pp. 287-328.

[4] H.T. Banks, R.C. Smith and Y. Wang, Smart Material Structures: Modeling, Estimation and Control, Masson/John Wiley, Paris/Chichester, 1996.

[5] W.G. Cady, Piezoelectricity, McGraw-Hill, New York, 1946.

[6] R.C.H. del Rosario and R.C. Smith, Spline approximation of thin shell dynamics, International Journal for Numerical Methods in Engineering, 40 (1997), pp. 2807-2840.

[7] R.C.H. del Rosario and R.C. Smith, LQR control of thin shell dynamics: formulation and numerical implementation, Journal of Intelligent Material Systems and Structures, 9(4) (1998), pp. 301-320.

[8] R.C.H. del Rosario and R.C. Smith, LQR Control of Shell Vibrations via Piezoceramic Actuators, International Series of Numerical Mathematics, 126, Birkhäuser, Basel (1998), pp. 247-265. 
[9] P. Ge and M. Jouaneh, Modeling Hysteresis in Piezoceramic Actuators, Precision Engineering, 17 (1995), pp. 211-221.

[10] P.K. Hansma, V.B. Elings, O. Marti and C.E. Bracker, Scanning Tunneling Microscopy and Atomic Force Microscopy: Application to Biology and Technology, Science, 242 (1988), pp. 209242.

[11] T. Ikeda, Fundamentals of Piezoelectricity, Oxford University Press, Oxford, 1990.

[12] E.L. Ince, Ordinary Differential Equations, Dover Publications, Inc., New York, 1956.

[13] A.W. Leissa, Vibration of Shells, NASA SP-288, 1973; Reprinted by the Acoustical Society of America through the American Institute of Physics, 1993.

[14] I.D. Mayergoyz, Mathematical Models of Hysteresis, Springer-Verlag, New York, 1991.

[15] J. Nealis and R.C. Smith, Partial Inverse Compensation Techniques for Linear Control Design in Magnetostrictive Transducers, Proceedings of the SPIE, Smart Structures and Materials 2001, Volume 4326, pp 462-473, 2001.

[16] P.M. Prenter, Splines and Variational Methods, Wiley, New York, 1975.

[17] R.C. Smith, A Domain wall model for asymmetric minor hysteresis loops in ferroelectric materials, to be submitted.

[18] R.C. Smith and C.L. Hom, Domain wall theory for ferroelectric hysteresis, Journal of Intelligent Material Systems and Structures, 10(3) (1999), pp. 195-213.

[19] R.C. Smith and Z. Ounaies, A domain wall model for hysteresis in piezoelectric materials, Journal of Intelligent Material Systems and Structures, 11(1) (2000), pp. 62-79.

[20] R.C. Smith and Z. Ounaies, A model for asymmetric hysteresis in piezoceramic materials, in Materials for Smart Systems III, Material Research Society Symposium Proceedings Volume 604 (2000), pp. 285-290.

[21] R.C. Smith, Z. Ounaies and R. Wieman, A Model for Rate-Dependent Hysteresis in Piezoceramic Materials Operating at Low Frequencies, Proceedings of the SPIE, Smart Structures and Materials 2000, Newport Beach, CA, Volume 3992 (2000), pp. 128-136.

[22] X. Tan, R. Venkataraman and P.S. Krishnaprasad, Control of Hysteresis: Theory and Experimental Results, Smart Structures and Materials 2001, Modeling, Signal Processing and Control in Smart Structures, SPIE Vol. 4326, (2001), pp. 101-112.

[23] G. Tao and P.V. Kokotović, Adaptive Control of Systems with Actuator and Sensor Nonlinearities, John Wiley and Sons, New York, 1996. 